\title{
MonoMax Suture: A New Long-Term Absorbable Monofilament Suture Made from Poly-4-Hydroxybutyrate
}

\author{
Erich K. Odermatt, ${ }^{1}$ Lutz Funk, ${ }^{2}$ Rainer Bargon, ${ }^{1}$ David P. Martin, ${ }^{3}$ \\ Said Rizk, ${ }^{3}$ and Simon F. Williams ${ }^{3}$ \\ ${ }^{1}$ Aesculap AG, Am Aesculap-Platz, 78532 Tuttlingen, Germany \\ ${ }^{2}$ B. Braun Surgical S.A., Carretera de Terrassa 121, 08191 Rubi, Spain \\ ${ }^{3}$ Tepha Inc., 99 Hayden Avenue, Lexington, MA 02421, USA
}

Correspondence should be addressed to Erich K. Odermatt, erich.odermatt@aesculap.de

Received 6 December 2011; Accepted 21 February 2012

Academic Editor: Kibret Mequanint

Copyright (C) 2012 Erich K. Odermatt et al. This is an open access article distributed under the Creative Commons Attribution License, which permits unrestricted use, distribution, and reproduction in any medium, provided the original work is properly cited.

\begin{abstract}
A long-term absorbable monofilament suture was developed using poly-4-hydroxybutyrate (P4HB) made from a biosynthetically produced homopolymer of the natural metabolite 4-hydroxybutyrate. The suture, called MonoMax, has prolonged strength retention. At 12 weeks, a size 3-0 MonoMax suture retains approximately 50\% of its initial tensile strength in vivo and is substantially degraded in one year with minimal tissue reaction. In contrast, PDS II monofilament suture (Ethicon, Inc., Somerville, NJ) has no residual strength in vivo after 12 weeks. In vivo, the MonoMax suture is hydrolyzed primarily by bulk hydrolysis, and is then degraded via the Krebs cycle. MonoMax is substantially more compliant than other monofilament sutures, and incorporates an element of elasticity. Its tensile modulus of $0.48 \mathrm{GPa}$ is approximately one-third of the value of the PDS II fiber providing an exceptionally flexible and pliable fiber with excellent knot strength and security. These features are further enhanced by the fiber's elasticity, which also improves knot security and may help prevent wound dehiscence. Because of its performance advantages, this suture may find clinical utility in applications where prolonged strength retention, and greater flexibility are required, particularly in procedures like abdominal wall closure where wound dehiscence is still a significant postsurgical complication.
\end{abstract}

\section{Introduction}

The development of synthetic absorbable sutures was one of the most important clinical advances in wound closure and suture technologies. When compared to catgut sutures, these synthetic alternatives typically have greater tensile strengths, more uniform degradation profiles, and a significantly reduced incidence of infection and tissue reaction. As a result, there has been widespread acceptance and use of synthetic absorbable sutures, and these products are now the most commonly used sutures in surgery and wound repair, where temporary wound support is needed.

In the early 1970s, suture manufacturers initially introduced synthetic absorbable sutures in a braided configuration made from polyglycolide (Dexon, Davis \& Geck) or copolymers of glycolide and lactide (Vicryl, Ethicon), because monofilament fibers of these materials were too stiff and difficult to handle [1]. However, braided synthetic absorbable sutures have a tendency to tear through tissue, and can potentiate infection via the interstices of their braided structure. To address these drawbacks, suture manufacturers initially developed coatings to reduce tissue drag, but in the 1980s introduced new polymers for use in monofilament sutures as a way to reduce the tissue drag and infection rates associated with the use of braided sutures. As a result, there is a broad range of synthetic, absorbable braided and monofilament sutures available today.

The first monofilament absorbable synthetic suture, PDS, a homopolymer of $p$-dioxanone, was introduced in 1984 by Ethicon (Somerville, NJ, USA) and eliminated some of the concerns associated with the use of braided sutures [1]. An improved version, PDS II, was later introduced 


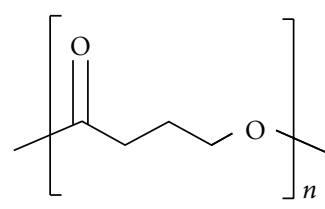

(a) poly-4-hydroxybutyrate

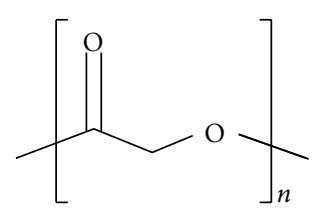

(b) polyglycolide

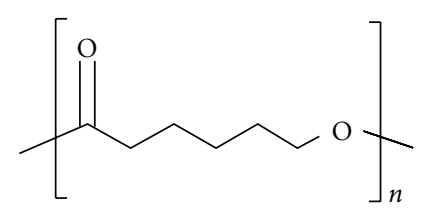

(c) polycaprolactone

Figure 1: Chemical structures of (a) poly-4-hydroxybutyrate (P4HB), (b) polyglycolide, and (c) $\varepsilon$-polycaprolactone.

by Ethicon. Other absorbable monofilaments have since been introduced, but in general the existing monofilament absorbable sutures do not handle as well as braids and typically require more knotting throws. Moreover, PDS II degrades relatively rapidly with a loss of approximately $50 \%$ of its initial strength 4-5 weeks after implantation making it unsuitable for use, where longer strength retention is required. Thus, there is still room to improve the performance of absorbable monofilament sutures.

To address the current shortcomings of existing absorbable monofilament sutures, a major research effort was initiated to develop a P4HB suture (MonoMax, B.Braun), the most pliable synthetic absorbable monofilament suture in clinical use. The suture is derived from a biosynthetic polyester known as poly-4-hydroxybutyrate (P4HB, Tepha, Inc.). This new absorbable polymer promises to be useful in a variety of medical device applications, but is seeing its first clinical utility as a monofilament suture. In this paper, the tensile strength, breaking strength retention, knot strength, in vitro and in vivo degradation, and biocompatibility of the $\mathrm{P} 4 \mathrm{HB}$ suture are described as well as the in vivo functionality of the MonoMax suture in an animal model for abdominal wall repair. Each of these parameters is critical in determining suture performance. Additional studies (not described herein) have also been undertaken to evaluate the clinical performance of the MonoMax suture in abdominal wall repair and demonstrate the superior performance of this new monofilament suture.

\section{Experimental Method}

2.1. Biosynthesis of Poly-4-Hydroxybutyrate. MonoMax Suture is derived from poly-4-hydroxybutyrate homopolymer, which is produced by Tepha, Inc. (Lexington, MA, USA) using a proprietary fermentation process [2]. Despite the polymer's biological method of production, the chemical structure of the polymer is very simple (Figure 1) and closely resembles the structure of existing synthetic absorbable biomaterials such as polyglycolide or poly-e-caprolactone. The polymer belongs to a larger class of materials called polyhydroxyalkanoates (PHAs) that are produced in nature by numerous microorganisms [3-5]. In nature these polyesters are produced as storage granules inside cells and serve to regulate energy metabolism (see [2-5] and references therein). They are also of commercial interest because of their thermoplastic properties, and relative ease of production from sustainable resources. While there has been considerable effort to develop other PHA materials for medical applications
$[3,4]$, P4HB is currently the only PHA cleared by the USFDA and $\mathrm{EU}$ regulatory agencies for use in clinical applications.

$\mathrm{P} 4 \mathrm{HB}$ is not naturally produced by any wildtype microorganism, but can be produced at scale for medical applications through biotechnology. Several biosynthetic routes are known to produce $\mathrm{P} 4 \mathrm{HB}[2,6,7]$. Notably, the chemical synthesis of P4HB has been attempted, but it is generally considered impossible to produce the polymer with sufficiently high molecular weight necessary for thermoplastic applications [8]. Recent theoretical calculations have tried to elucidate the mechanistic reason for the difficulty in synthesizing P4HB [9]. Tepha, Inc. produces P4HB utilizing a genetically engineered Escherichia coli K12 microorganism that incorporates new biosynthetic pathways to produce the homopolymer. The polymer accumulates as distinct granules inside the cells during fermentation and can be extracted at the end of the process in a very pure form. Typically, yields of $\mathrm{P} 4 \mathrm{HB}$ exceed 50 grams per liter of fermentation broth in less than 48 hours making large-scale production attractive. The ${ }^{1} \mathrm{H}$-NMR spectrum of purified P4HB shows three characteristic peaks that are consistent with the structure for the polymer $\left({ }^{1} \mathrm{H}-\mathrm{NMR}(400 \mathrm{MHz}, \mathrm{CDCl} 3) \delta=1.94(\mathrm{~m}\right.$, $2 \mathrm{H}), 2.37(t, 2 \mathrm{H}, J=7.4 \mathrm{~Hz}), 4.10(t, 2 \mathrm{H}, J=6.6 \mathrm{~Hz}))$. No extraneous peaks were found that would otherwise indicate the presence of soluble organic contaminants in the sample or other monomeric constituents in the polymer.

The weight average molecular weight $\left(M_{w}\right)$ for the polymer was found to be $400-600 \mathrm{kDa}$ as measured by gel permeation chromatography (GPC) in chloroform relative to polystyrene standards of narrow polydispersity. This $M_{w}$ is well suited to melt spinning of the high-strength monofilament suture fibers. Endotoxin content of the purified polymer, measured by the Limulus amebocyte lysate (LAL) assay, is very low and is well below the requirements established for medical devices.

2.2. Preparation of the MonoMax Suture. Poly-4-hydroxybutyrate polymer was pelletized and melt extruded into monofilaments, quenched, and drawn in line with stretch ratios of 6 to $11 \mathrm{x}$ in a multistage drawing process in order to form the suture fiber and achieve the desired tensile and handling properties of the MonoMax suture.

2.3. Sterilization of the MonoMax Suture. Monofilament fibers of the MonoMax suture were cut to uniform lengths, packaged in ethylene oxide sterilization bags and exposed to a cold ethylene oxide (EtO) sterilization cycle. Sterility was confirmed by testing random samples by direct transfer 
to test media per USP standard test method $\langle 25\rangle$. The EtO sterilization cycle was subsequently validated. EtO residuals (EtO, epichlorohydrine ECH, and ethylene glycol EG) were found to be below 2 ppm after the aeration [10].

2.4. Mechanical Properties of the MonoMax Suture. The tensile properties of sterile monofilament fibers from multiple lots were determined using a universal mechanical tester according to procedures described in the US Pharmacopeia (USP) standard for testing tensile properties of surgical sutures (USP $\langle 881\rangle$ ) [11]. Suture diameter was determined in accordance with the USP standard by making three measurements at the two ends and middle of the sample, except for explanted samples, where diameter was determined by averaging measurements from both ends of the sample. This was done to prevent damaging the middle section of explanted samples prior to tensile testing. The tensile properties of the monofilament samples were determined using either an Instron 3535 or a Zwick Z005 universal testing machine equipped with pneumatic fiber grips using a gauge length of $200 \mathrm{~mm}$, and a strain rate of $300 \mathrm{~mm} /$ minute. During testing, the location of the break was recorded. The specimen was rejected if the break occurred at the grips (i.e., outside the gauge length). Values for straight tensile strength, Young's modulus, elongation-to-break, and knot tensile strength were determined. The latter value was determined by following the procedure described in the European Pharmacopeia [12]. The bending stiffness of the P4HB suture was measured using a Frank 56585 bending stiffness testing device, applying a speed of determination of $6^{\circ} \%$ ( max. angle $30^{\circ}$; holding time $2 \mathrm{sec}$.), a measuring length of $1.0 \mathrm{~mm}$, and a preforce of $1 \mathrm{mN}$.

2.5. In Vitro Degradation of the MonoMax Monofilament. Sterilized P4HB monofilament suture fibers of size 3-0 were incubated in Dulbeco's phosphate-buffered saline ( $\mathrm{pH} 7.4$; $37^{\circ} \mathrm{C}$ ) to determine the stability of the monofilament under these conditions. The results were compared to control samples of the same fiber lot tested at the beginning of the study without any preliminary treatment in buffer solution. Sodium azide $(0.05 \% \mathrm{wt} / \mathrm{vol})$ was included in the buffer as a biocide. Samples were removed at $t=1,2,3,4,6,8$, 12,16 , and 20 weeks after incubating and tested for tensile strength. Additionally, the stability of the $\mathrm{P} 4 \mathrm{HB}$ suture was determined in an accelerated model of in vitro degradation. The suture was incubated in $3 \mathrm{M}$ hydrochloric acid at $37^{\circ} \mathrm{C}$. Samples were removed for straight tensile testing and $M_{w}$ determinations after 5, 24, and 48 hours incubation. To image the morphology of a fiber after accelerated in vitro degradation, the suture was incubated in a less concentrated $2 \mathrm{M}$ aqueous $\mathrm{HCl}$ solution for 7 days, in order to capture the degradation process in more detail.

2.6. In Vivo Breaking Strength Retention of the MonoMax Monofilament Suture after Ethylene Oxide Sterilization. A subcutaneous implantation study in rabbits was used to compare the breaking strength retention of the MonoMax suture to PDS II suture (Ethicon, Inc.) as a control.
Strength retention of the sutures was determined at 1, 2, $3,4,5,6,12,16$, and 20 weeks after the implantation date. A total of eighteen SPF albino rabbits (New Zealand White, Charles River Deutschland GmbH, weight 2-3 kgs) were arbitrarily assigned to the termination intervals, two rabbits per each time point. In preparation for the surgery, the fur was clipped from the area over the paravertebral muscles and the back scrubbed with 70\% ethyl alcohol. The rabbits were anesthetized by an intramuscular injection of ketamine and xylazine applying a dosage of $40 \mathrm{mg} / \mathrm{kg}$ (ketamine) and $6 \mathrm{mg} / \mathrm{kg}$ (xylazine). Before the implantation, packages containing reels of ethylene oxide sterilized size 3-0 MonoMax suture were opened on a sterile field and strands of approximately $150 \mathrm{~cm}$ length were cut from the reels. Small coils were made from the sutures in order to implant them later into the subcutaneous tissue. The same procedure was repeated using $90 \mathrm{~cm}$ stands of PDS II as reference samples. After making 3 small lateral incisions $3 \mathrm{~cm}$ in length on both sides of the spinal column, small subcutaneous pockets were formed, and the suture coils were placed inside the pockets. MonoMax fibers were implanted on the left, while PDS II control samples were implanted on the right side of the spinal column. The skin was closed with atraumatic nonabsorbable suture material (Premilene, B. Braun) and finally sealed with a tissue adhesive (Histoacryl, B. Braun). At appropriate intervals, the designated rabbits were euthanized by an intravenous injection of $0.3 \mathrm{~mL} / \mathrm{kg}$ T61. The skin of the rabbits was opened by a longitudinal incision along the spinal column, and extreme care was used during the dissection to avoid damaging the implants. The three MonoMax suture samples and three PDS II control suture samples from each animal were explanted, put in prelabeled containers and prepared for the tensile strength measurements. Straight pull tensile strengths and knot pull strengths of the explanted suture fibers were determined as described above according to USP $\langle 881\rangle$ [11]. After tensile testing, the remaining fiber fragments were retained for $M_{w}$ analysis by GPC (Polymer Standards Service GmbH, Mainz, Germany). The GPC measurements were conducted using chloroform as eluent and calibrating against polystyrene standards.

2.7. In Vitro Knotting Studies. To evaluate the knot security of the suture, the following in vitro test procedure was developed to simulate the real-life behavior of a surgeon performing a knot. For this test, the suture was tied around a plastic tube with a diameter of $9 \mathrm{~cm}$, and the two ends of the strand were knotted tightly with a surgical knot $(2=1)$ to form a loop according to Tera and Aberg [13]. After making the knot, the suture loop was cut on the side opposite the position of the knot. This results in a suture strand with a $2=1$ knot called surgical knot in the middle (see Figure 2).

Both fiber ends were clamped in a tensile testing machine and the knotted strand was pulled apart with a traverse speed of $100 \mathrm{~mm} / \mathrm{min}$. If the suture breaks at the knot for 10 samples, the knot security factor (KSF) is defined as KSF = 0 . If the knot slips and unties when applying a force for one or more samples out of ten, then another ten samples are prepared and tested as above but with an additional 


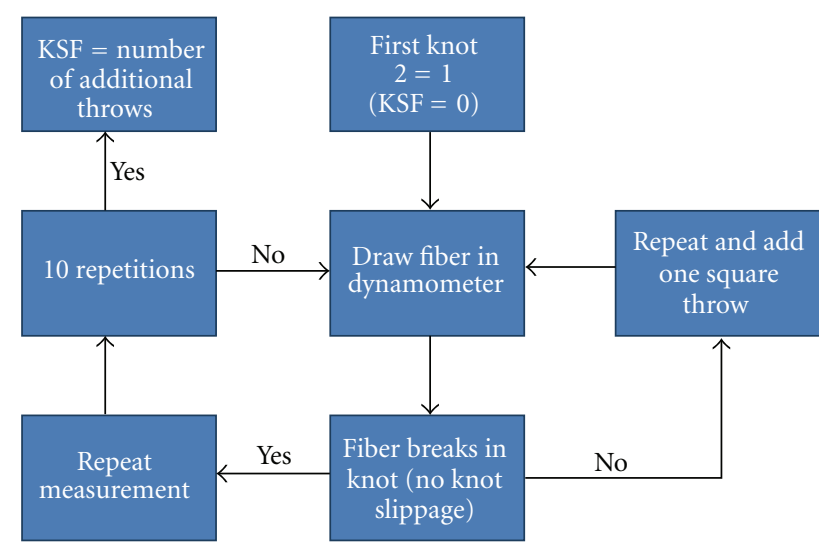

FIGURE 2: Schematic drawing of the knot security factor procedure.

square knot. The knot security factor (KSF) increases by 1 for each additional throw needed on top of the first surgical knot $(2=1)$. The KSF is then defined as the number of additional throws needed to prevent all ten knotted samples from untying when an external force is applied. A knot security factor (KSF) of 2-4 proves to be excellent for a monofilament.

2.8. Biocompatibility Testing. The testing described in this section was performed to establish the biocompatibility of the ethylene oxide sterilized MonoMax suture in accordance with the International Standard ISO-10993 "Biological Evaluation of Medical Devices Part-1: Evaluation and Testing" [14]. Biocompatibility testing was performed at NAMSA (Ohio) and consisted of the tests for: cytotoxicity, irritation and sensitization, systemic toxicity, genotoxicity, carcinogenicity and reproductive toxicity, interactions with blood, and the local effects after implantation.

\subsection{Histological Evaluation of MonoMax Suture Degradation.} To examine the mass absorption process and the tissue reaction involved in the degradation process, ethylene oxide and gamma radiation sterilized MonoMax sutures were implanted in the paravertebral muscles of white SPF-Wistar rats (Dr. Schreiter, Freytox GmbH, Osteroda, Germany). A total of 56 rats were implanted with size 3-0 MonoMax sutures using a mattress stitch so that $2 \mathrm{~cm}$ of material was placed in the muscle. To distinguish between the different test objects, the EtO sterilized sutures were implanted to the left of the vertebral column, whereas the gamma sterilized sutures were placed on the right side. Prior to the implantation in the muscle, the Wistar rats were anaesthetized using $80 \mathrm{mg}$ ketamine and $12 \mathrm{mg}$ xylazine per $\mathrm{kg}$ of bodyweight, clipped free of fur on the back, and the skin was disinfected with $70 \%$ ethanol. The skin was opened with a $4 \mathrm{~cm}$ median incision along the vertebral column and the subcutaneous tissue as well as the paravertebral muscle were prepared. After implantation, the skin incision was closed by a subcutaneous suture and sealed using a tissue adhesive (Histoacryl, BBraun). The animals were distributed to termination dates according to the scheme in Table 1.
TABLe 1: Implantation scheme of size 3-0 P4HB sutures in the paravertebral muscle of SPF-Wistar rats.

\begin{tabular}{|c|c|c|}
\hline \multicolumn{3}{|c|}{ Implantation scheme MonoMax USP 3-0 } \\
\hline Group & Time of termination & Number of animals \\
\hline 1 & 4 weeks p.op. & 5 \\
\hline 2 & 8 weeks p.op. & 5 \\
\hline 3 & 12 weeks p.op. & 5 \\
\hline 4 & 16 weeks p.op. & 5 \\
\hline 5 & 24 weeks p.op. & 6 \\
\hline 6 & 32 weeks p.op. & 6 \\
\hline 7 & 37 weeks p.op. & 6 \\
\hline 8 & 42 weeks p.op. & 6 \\
\hline 9 & 50 weeks p.op. & 6 \\
\hline 10 & 64 weeks p.op. & 6 \\
\hline
\end{tabular}

p.op.: post operation.

At the appropriate termination dates, the animals were euthanized by $\mathrm{CO}_{2}$ inhalation, the paravertebral muscle tissue removed entirely, labeled precisely, fixed in $4 \%$ neutral buffered formalin (NBF) and histologically processed (embedded, sectioned and stained in hematoxylin and eosin) for macroscopic, and microscopic evaluation by a qualified pathologist (Dr. Weber, Labor für Veterinärmedizinische Diagnostik, Bad Salzungen, Germany).

2.10. Surgical Functionality. The performance and surgical functionality of the MonoMax suture was evaluated in a rabbit model of soft tissue approximation. This study was performed at the animal research facilities of the FriedrichSchiller-University in Jena, Germany by Dr. Ulrich Stock. Full-thickness abdominal incisions were repaired with either MonoMax suture or PDS II sutures (Ethicon, Inc.) as a comparative control. Eighteen (18) healthy adult New Zealand white rabbits (weighing $2.5-4 \mathrm{~kg}$ ) were used in the evaluation. Following intramuscular induction and maintenance using intravenous anesthesia, the abdominal implant sites were clipped free of fur. Under sterile conditions, an abdominal midline skin incision of $4 \mathrm{~cm}$ length was made, and the skin spread using a self-retainer. Two paramedian muscular and peritoneal incisions ( $3 \mathrm{~cm}$ length) were made and closed either with running 3/0 PDS II or running 3/0 MonoMax sutures (see Figure 9). After thorough control for hemostasis, the skin was closed using absorbable Vicryl sutures. Special care was taken to ensure that the distance from the skin closure to the study suture implant site was at least $2 \mathrm{~cm}$. Each rabbit was implanted with one test and one control suture. Animals were maintained for periods of 4,8 , and 12 weeks ( 6 rabbits per time point) and observed daily to ensure proper healing of the implant sites. At the end of the appropriate time points, the animals were weighed and sacrificed by an injectable barbiturate and tissue sections containing the implanted sutures were excised from the animals. Gross observations were made for hernias as indicators of weakening sutures and adhesions. 
TABLE 2: Comparison of tensile properties of size 1 absorbable suture materials.

\begin{tabular}{llcccccccccc}
\hline Suture & $\begin{array}{l}\text { Chemical } \\
\text { composition }\end{array}$ & $\begin{array}{c}\text { Diameter } \\
x_{\text {ave }}(\mathrm{mm})\end{array}$ & s.d. & $\begin{array}{c}\text { LPTS } \\
(\mathrm{N})\end{array}$ & s.d. & $\begin{array}{c}\text { KPTS } \\
(\mathrm{N})\end{array}$ & s.d. & $\begin{array}{c}\text { Bending } \\
\text { stiffness (m) }\end{array}$ & s.d. & $\begin{array}{c}\text { Young's } \\
\text { modulus (GPa) }\end{array}$ & s.d. \\
\hline MonoMax & $\begin{array}{l}\text { Poly-4- } \\
\text { hydroxybutyrate }\end{array}$ & 0,517 & 0,007 & 118 & 6,1 & 74 & 3,1 & 363 & 7 & 0,485 & 0,01 \\
PDS II & $\begin{array}{l}\text { Poly-p- } \\
\text { dioxanone }\end{array}$ & 0,532 & 0,008 & 109 & 4,9 & 63 & 5,2 & 753 & 72 & 1,370 & 0,03 \\
Monocryl & $\begin{array}{l}\text { Polyglecaprone } \\
\text { 25 }\end{array}$ & 0,531 & 0,002 & 183 & 7,3 & 92 & 5,9 & 532 & 21 & 0,725 & 0,03 \\
Biosyn & Glycomer 631 & 0,538 & 0,005 & 176 & 24,9 & 89 & 10,5 & 679 & 64 & 0,838 & 0,03 \\
Maxon & Polyglyconate & 0,550 & 0,006 & 177 & 8,2 & 118 & 9,3 & 794 & 41 & 0,941 & 0,06 \\
\hline
\end{tabular}

TABLE 3: Knot security factor (KSF) and knot pull tensile strength determination for MonoMax sutures size 1 to 3-0.

\begin{tabular}{lccc}
\hline Size & KSF & Ave. KPTS $(\mathrm{N})$ & s.d. \\
\hline 1 & 3 & 45,4 & 3,4 \\
0 & 3 & 42,4 & 5,0 \\
$2-0$ & 2 & 28,4 & 3,2 \\
$3-0$ & 2 & 20,9 & 2,2 \\
\hline
\end{tabular}

\section{Results}

3.1. Mechanical Properties. The tensile properties of the MonoMax suture size 1 are compared with those of PDS II, Monocryl, Biosyn [15], and Maxon [16] sutures in Table 2.

As is evident from Table 2, the size 1 MonoMax suture is stronger than the other homopolymer suture, namely, PDS II. However, these homopolymer sutures are not as strong as the copolymer sutures, which are made of an ABA block copolymer type structure with two glycolide hard segments. It should be noted that the MonoMax suture has the smallest diameter of all the sutures tested. This was done to keep MonoMax sizing close to the USP specification for diameter. In addition to offering a high tensile strength, the MonoMax suture is very flexible. It has the lowest Young's modulus value of the current commercial monofilament sutures and its bending stiffness is about half that of the other sutures listed in Table 2. The bending stiffness of Monocryl is the lowest of the competitive sutures, but is still 1.5 fold that of the MonoMax suture. The lower values of modulus and bending stiffness are important properties of the MonoMax suture that provide the suture with good handling for surgical suturing and knot tying, and improved flexibility and pliability compared to current commercial offerings. Besides flexibility, a smooth surface is also important for knotting to allow for knot run down and repositioning during surgical usage. The combination of the smooth surface with the flexibility of the MonoMax suture provides a suture with low tissue drag that minimizes trauma to the sutured tissues. The smooth, nonporous surface of monofilaments like the MonoMax suture can also reduce the chance of infection due to the capillary effect that can be caused by the interstices present in braided, multifilament sutures.

3.2. Knot Security of the MonoMax Monofilament. Due to their smooth surface, some surgeons have raised concerns about the knot security of monofilament sutures relative to braided sutures. Therefore, many surgeons traditionally use up to 6 throws to tie a secure knot when suturing with a monofilament. We performed knot security tests with size 1 MonoMax material as described in Section 2.7, tying a surgical knot $2=1$ [13]. A knot security factor was determined by accounting for every additional square knot needed to secure the knot. Due to the soft and interlocking surface of the MonoMax suture, a total of only 4-5 throws $(2=1=1=1 ; 2=1=1=1=1)$ was enough to tie a secure knot, corresponding to a knot security factor of 2 to 3 (see Table 3).

The tensile strength of the fiber after performing the KSF test is significantly lower than the knot pull tensile strength measured for the respective sizes of the MonoMax suture according to the US Pharmacopeia $\langle 881\rangle$ [11]. The reason for this behavior lies in the application of multiple knots (KSF method) instead of making only one single knot which leads to additional frictional forces lowering the strength of the fiber.

3.3. In Vitro Strength Retention of MonoMax Monofilament. The breaking strength retention of an absorbable suture is defined as:

$$
\operatorname{BSR}(\%)=\frac{\text { actual strength }}{\text { initial strength }} * 100 \% \text {. }
$$

Figure 3 shows the in vitro knot pull tensile strength (KPTS) retention of size 3-0 MonoMax and PDS II suture in Dulbeco's phosphate-buffered saline $\left(\mathrm{pH} 7.4 ; 37^{\circ} \mathrm{C}\right)$ for a period of 20 weeks.

The size 3-0 MonoMax monofilament shows highstrength retention during the first 12 weeks of the incubation when the overall strength remains nearly unchanged. This period is followed by a decrease of breaking strength retention to $20 \%$ to $30 \%$ of the suture's initial strength until week 20, which is attributed to a slow hydrolysis of the polymer. In contrast, PDS II suture shows a more steady decrease of its KPTS, retaining an average value of $51 \%$ of its initial strength after 8 weeks and losing almost all of its tensile strength after 12 weeks, when only $7 \%$ of the strength is left.

When comparing the absolute knot pull tensile strength of the size 3-0 suture materials, the initial KPTS of MonoMax suture $(36.2 \mathrm{~N})$ is significantly higher than for PDS II 


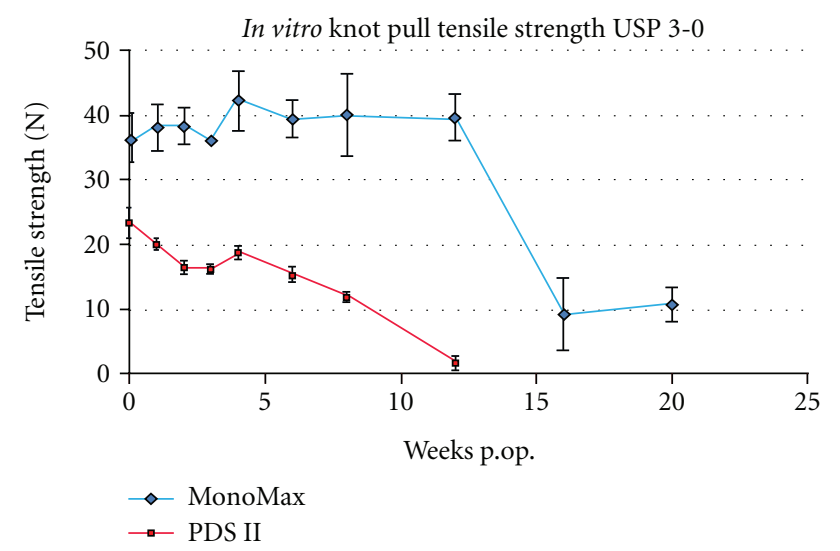

Figure 3: Knot pull tensile strength degradation profile of a $\mathrm{P} 4 \mathrm{HB}$ monofilament fiber of size 3-0 incubated in Dulbecco's phosphate buffered saline $\left(\mathrm{pH} 7.4 ; 37^{\circ} \mathrm{C}\right)$.

$(23.3 \mathrm{~N})$, which only has about $64 \%$ of the initial strength of MonoMax suture (see Table 4).

Under the highly acidic conditions of the accelerated in vitro testing $\left(3 \mathrm{M} \mathrm{HCl}, 37^{\circ} \mathrm{C}\right)$, the MonoMax suture undergoes a much more rapid loss of strength (see Figure 4).

These extreme conditions are meant as a rapid bench test for comparing different lots of material, rather than being representative of physiologic conditions. Within 48 hours, the linear breaking strength of MonoMax size 3-0 decreases to about $15 \%$ of its initial value under the afore-mentioned conditions. Figures 5(a) and 5(b) show a scanning electron micrograph (SEM) of the MonoMax suture before and after a similar accelerated in vitro degradation in $2 \mathrm{M} \mathrm{HCl}$ at $37^{\circ} \mathrm{C}$ solution for 7 days showing an advanced state of degradation caused by the hydrolytic digestion of the suture material. Figures 5(c) and 5(d) show SEM images of the MonoMax suture after 12 weeks in vivo. During this time period it is evident that the MonoMax suture is degrading by surface erosion as well as bulk hydrolysis that is shown through $M_{w}$ analysis.

3.4. In Vivo Strength Retention of the MonoMax Monofilament. The in vivo knot pull tensile strength (KPTS) retention of size 3-0 MonoMax suture in a rabbit model is shown in Figure 6 and in more details in Table 5 and is compared with PDS II suture of the same size as a control.

As can be seen in Figure 6 and Table 5, the MonoMax suture retains its strength significantly longer in vivo than the PDS II suture. At 8 to 12 weeks, the size 3-0 MonoMax suture still retains approximately $50 \%$ of its initial knot pull tensile strength, whereas PDS II retains 50\% after 4 to 6 weeks. Furthermore, PDS II does not provide any tensile strength to the sutured tissue after 12 weeks p.op. The MonoMax monofilament, however, still has about $20 \%$ to $30 \%$ of its initial strength at 20 weeks after implantation. As mentioned before in the in vitro testing (phosphate buffer), the absolute breaking strength values of MonoMax suture after 20 weeks correspond to $46 \%$ of the initial breaking strength of PDS II because of the superior initial breaking strength of the MonoMax suture. As shown in Figure 7, the

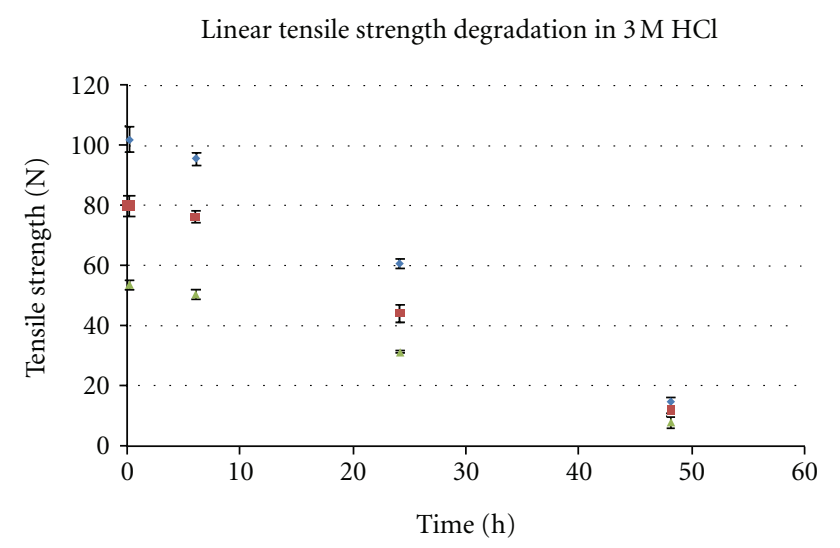

\footnotetext{
- MonoMax USP 1

- MonoMax USP 0

- MonoMax USP 2-0
}

Figure 4: Accelerated in vitro degradation profile of a $\mathrm{P} 4 \mathrm{HB}$ monofilament fiber size USP 1, USP 0, and USP 2-0 incubated in hydrochloric acid $\left(3 \mathrm{M} ; 37^{\circ} \mathrm{C}\right)$.

molecular weight of the MonoMax monofilament gradually decreases in vivo from an initial value of $M_{w} 286,000 \mathrm{Da}$, and the suture is substantially degraded within one year (see Section 3.6).

3.5. Biocompatibility of MonoMax Sutures. The biocompatibility of the P4HB suture was found to be excellent. Test results are shown in Table 6 . The suture passed all tests with no evidence of toxicity.

The MonoMax suture is derived biosynthetically from the natural metabolite 4-hydroxybutyrate. This monomer is a normal constituent of the mammalian blood serum that has been extensively studied and is known to occur within a variety of tissues, including brain, heart, kidney, liver, lung, muscle, and brown fat [17]. The concentration of the monomer in these tissues ranges from 2.3 to $37.4 \mathrm{nmoles} / \mathrm{g}$ of tissue (or 0.24 to $3.8 \mathrm{mg} / \mathrm{kg}$ of tissue, based on a formula weight of $104 \mathrm{~g} / \mathrm{mol}$ for the monomer). The body quickly metabolizes 4-hydroxybutyrate via the Krebs cycle to carbon dioxide and water and its half-life is just 35 minutes [18]. Two metabolic precursors to the 4-hydroxy-butyrate monomer, namely 1,4-butanediol and gamma-butyrolactone, have both been thoroughly studied in two NIH sponsored National Toxicology Program (NTP) studies that evaluated their toxicology, genotoxicity, and carcinogenesis $[19,20]$. Both precursors were found to lack toxic and carcinogenic potential, and the NTP concluded that 4-hydroxybutyrate also lacks toxic and carcinogenic potential because of the rapid and complete conversion of the precursors to this metabolite. Prior to this study, however, the biocompatibility of the poly-4-hydroxybutyrate homopolymer had not been fully evaluated in vivo. The results of the studies on the polymer are reported in Table 6.

3.6. Histology. The tissue surrounding the implanted MonoMax monofilament and PDS II control sutures was analyzed 
TABLE 4: Results of the in vitro degradation of the knot pull tensile strength of USP 3-0 MonoMax and PDS II sutures in phosphate buffer at pH 7.4.

\begin{tabular}{|c|c|c|c|c|c|c|c|c|c|c|}
\hline \multirow[b]{2}{*}{ Weeks } & \multicolumn{5}{|c|}{ MonoMax USP 3-0 } & \multicolumn{5}{|c|}{ PDS II USP 3-0 } \\
\hline & $x_{\text {ave }}[\mathrm{N}]$ & s.d. & $x_{\min }[\mathrm{N}]$ & $x_{\max }[\mathrm{N}]$ & BSR [\%] & $x_{\text {ave }}[\mathrm{N}]$ & s.d. & $x_{\min }[\mathrm{N}]$ & $x_{\max }[\mathrm{N}]$ & BSR [\%] \\
\hline 0 & 36,2 & 3,81 & 29,4 & 42,1 & 100 & 23,3 & 2,33 & 20,5 & 27,2 & 100 \\
\hline 1 & 38,1 & 3,55 & 32,1 & 42,7 & 105 & 20,1 & 0,95 & 18,5 & 21,7 & 86 \\
\hline 2 & 38,4 & 2,86 & 33,3 & 43,1 & 106 & 16,5 & 0,92 & 15,4 & 18,2 & 71 \\
\hline 3 & 36,2 & 4,13 & 28,2 & 41,3 & 100 & 15,9 & 0,64 & 15,2 & 17,2 & 68 \\
\hline 4 & 42,2 & 4,61 & 32,6 & 48,0 & 116 & 18,7 & 0,90 & 17,2 & 20,1 & 80 \\
\hline 6 & 39,5 & 2,84 & 33,7 & 44,1 & 109 & 14,8 & 1,10 & 13,6 & 16,5 & 63 \\
\hline 8 & 40,0 & 6,37 & 25,4 & 51,2 & 110 & 11,9 & 0,85 & 10,7 & 13,3 & 51 \\
\hline 12 & 39,7 & 3,49 & 32,4 & 41,6 & 104 & 1,7 & 0,38 & 1,1 & 2,2 & 7 \\
\hline 16 & 9,2 & 5,55 & 3,2 & 16,7 & 25 & n.d. & n.d. & n.d. & n.d. & n.d. \\
\hline 20 & 10,7 & 2,57 & 8,3 & 16,2 & 29 & n.d. & n.d. & n.d. & n.d. & n.d. \\
\hline
\end{tabular}

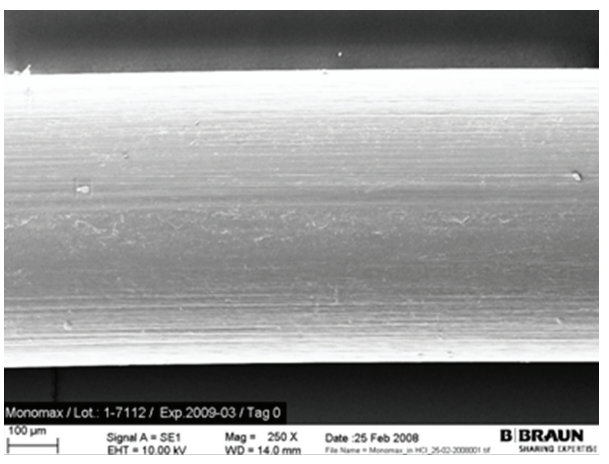

(a) P4HB fiber $t=0 \mathrm{~d}$

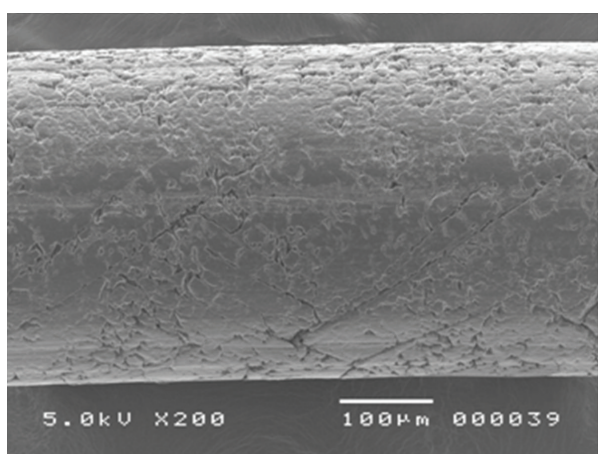

(c) P4HB fiber 12 weeks p.op.

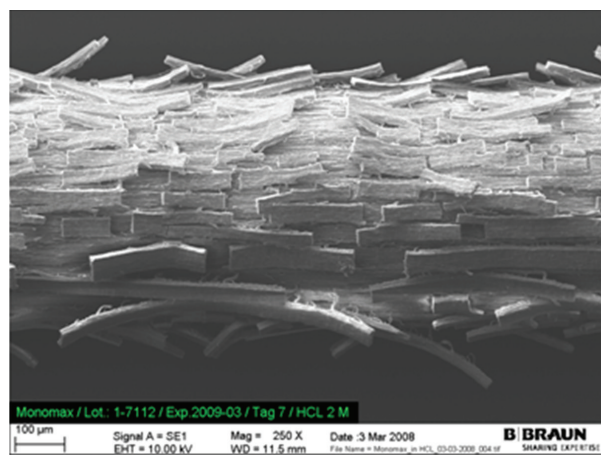

(b) P4HB fiber $t=7 \mathrm{~d}$

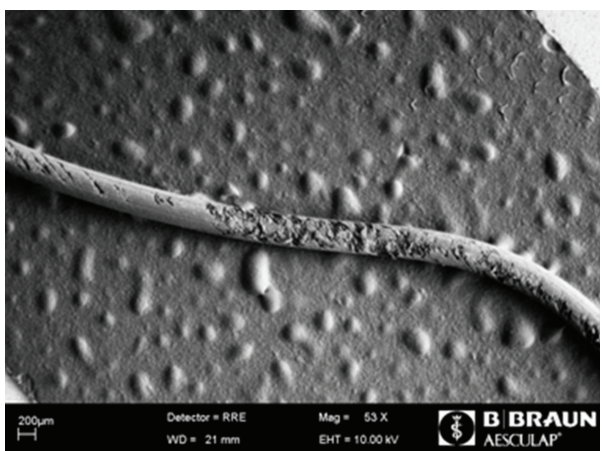

(d) P4HB fiber 12 weeks p.op.

FIgURE 5: SEM photomicrograph showing the surface of a P4HB monofilament fibers: (a) P4HB fiber size 1 before incubation in $2 \mathrm{M}$ HCl, (b) after incubation in $2 \mathrm{M} \mathrm{HCl}$ for $7 \mathrm{~d}$, (c) and (d) P4HB monofilament size 3-0 after being implanted subcutaneously in rabbits for 12 weeks.

for a tissue reaction at $4,8,12,16,24,32,37,42,50$, and 64 weeks. Formalin-fixed tissue samples from each test animal were sectioned and assessed by a board certified pathologist for the following: inflammation, fibrosis, hemorrhage, necrosis, degeneration, foreign debris, and relative size of the involved area. Histopathological evaluation showed that there were no significant indications of a local toxic effect for the MonoMax suture. Ethylene oxide and gamma-sterilized MonoMax fiber samples showed similar histological behavior at the examined termination intervals. After 8 weeks p.op., the tissue reaction was characterized primarily by the presence of mononuclear cells (macrophages, lymphocytes, and plasma cells) involved in the digestion of the capsule present around the fiber. Almost no granulocytic reaction was present at that time point and the suture fiber appeared intact (see Figure 8(a)). Histological signs of digestion of the fiber by mononuclear cells (macrophages) appear at 24 weeks. At this time, degradation is apparent at the surface of the fiber. The 32 weeks p.op. results show decomposition of the fiber structure and phagocytosis by macrophages, 
TABLE 5: Results of the knot pull tensile strength (KPTS) in vivo degradation of USP 3-0 MonoMax and PDS II sutures subcutaneously implanted in the subcutis of rabbits.

\begin{tabular}{lcccccccccc}
\hline & \multicolumn{4}{c}{ MonoMax USP 3/0 } & \multicolumn{4}{c}{ PDS II USP 3/0 } \\
Weeks p.op. & $x_{\text {ave }}[\mathrm{N}]$ & s.d. & $x_{\min }[\mathrm{N}]$ & $x_{\max }[\mathrm{N}]$ & BSR [\%] & $x_{\text {ave }}[\mathrm{N}]$ & s.d. & $x_{\min }$ & $x_{\max }$ & BSR \\
\hline 0 & 36,2 & 3,81 & 29,4 & 42,1 & 100 & 23,3 & 2,3 & 20,5 & 27,2 & 100 \\
1 & 37,0 & 2,17 & 34,5 & 41,6 & 102 & 22,6 & 1,1 & 20,8 & 24,2 & 97 \\
2 & 34,5 & 3,05 & 29,4 & 39,3 & 95 & 19,1 & 4,3 & 8,3 & 22,4 & 82 \\
3 & 36,9 & 5,83 & 23,3 & 43,6 & 102 & 16,6 & 1,3 & 14,7 & 18,8 & 71 \\
4 & 30,0 & 7,80 & 20,6 & 44,8 & 83 & 15,8 & 1,5 & 13,9 & 18,8 & 68 \\
6 & 20,8 & 8,78 & 3,5 & 34,3 & 57 & 11,5 & 1,3 & 8,9 & 12,8 & 49 \\
8 & 22,1 & 4,94 & 15,0 & 30,2 & 61 & 7,1 & 1,9 & 2,8 & 8,8 & 30 \\
12 & 22,1 & 6,31 & 11,8 & 33,3 & 61 & n.d. & n.d. & n.d. & n.d. & n.d. \\
16 & 9,2 & 5,55 & 3,0 & 16,7 & 25 & n.d. & n.d. & n.d. & n.d. & n.d. \\
20 & 10,7 & 2,57 & 7,8 & 16,2 & 29 & n.d. & n.d. & n.d. & n.d. & n.d. \\
\hline
\end{tabular}

TABle 6: Biocompatibility Testing Performed on the P4HB Suture Fiber.

\begin{tabular}{|c|c|c|c|}
\hline Tests For & Method & Results & Remark \\
\hline Cytotoxicity & MEM elution & Passed & No evidence of cell lysis or toxicity \\
\hline Cytotoxicity & Agarose overlay & Passed & No evidence of cell lysis or toxicity \\
\hline Irritation and sensitization & $\begin{array}{l}\text { Intracutaneous reactivity with saline and } \\
\text { sesame oil extracts }\end{array}$ & Passed & No evidence of significant irritation \\
\hline Irritation and sensitization & $\begin{array}{l}\text { Maximization sensitization with saline } \\
\text { and sesame oil extracts }\end{array}$ & Passed & $\begin{array}{l}\text { No evidence of delayed dermal } \\
\text { sensitization }\end{array}$ \\
\hline Systemic toxicity & $\begin{array}{l}\text { Acute systemic toxicity with saline and } \\
\text { sesame oil extracts }\end{array}$ & Passed & No evidence of systemic toxicity \\
\hline Systemic toxicity & Material-mediated pyrogenicity & Passed & Nonpyrogenic \\
\hline $\begin{array}{l}\text { Genotoxicity, carcinogenicity, } \\
\text { and reproductive toxicity }\end{array}$ & $\begin{array}{l}\text { Bacterial reverse mutation study with } \\
\text { saline and ethanol extracts }\end{array}$ & Passed & Nonmutagenic \\
\hline $\begin{array}{l}\text { Genotoxicity, carcinogenicity, } \\
\text { and reproductive toxicity }\end{array}$ & Chromosomal aberration in $\mathrm{CHO}$ cells & Passed & Not genotoxic \\
\hline $\begin{array}{l}\text { Genotoxicity, carcinogenicity, } \\
\text { and reproductive toxicity }\end{array}$ & $\begin{array}{l}\text { Mouse micronucleus study with saline } \\
\text { and sesame oil extracts }\end{array}$ & Passed & $\begin{array}{l}\text { Not genotoxic. No evidence of } \\
\text { cellular toxicity. }\end{array}$ \\
\hline Local effects after implantation & Subchronic implantation & Passed & Nonirritant \\
\hline Local effects after implantation & Chronic implantation & Passed & Nonirritant \\
\hline Interaction with blood & In vitro hemolysis with saline extract & Passed & Nonhemolytic \\
\hline
\end{tabular}

with the macrophages penetrating the whole fiber crosssection. The fiber material appears amorphous and swollen (see Figure 8(c)). The histology images after 50 weeks p.op. indicate a complete loss of the initial fiber structure. The voids are filled with a fine vacuolar, granular material, and cellular reaction was present at the implantation site. No granulocytic reaction was detectable and all histological samples showed low tissue reaction intensity. After 64 weeks p.op. (see Figure $8(\mathrm{~d})$ ), no fiber could be detected in two of the four animals. The samples recovered from the other two animals had similar histological findings like those of the 50 week p.op. group exhibiting loss of fiber structure and a vacuolar, granular material replacing the suture.

Summarizing the results, the MonoMax monofilament size 3-0 shows a good biocompatibility as well as a very low tissue reaction throughout the whole study. After 24 weeks, the MonoMax suture undergoes a progressive phagocytosis with the participation of macrophages resulting in a complete loss of the fiber structure after 50 weeks p.op., when the fiber is replaced by a fine vacuolar, granular material. Finally, the MonoMax monofilament is essentially absorbed after 64 weeks.

3.7. Surgical Functionality of MonoMax Monofilament. The MonoMax suture was evaluated in a rabbit model of abdominal soft tissue approximation with PDS II as a control (see Figure 9).

All the animals $(n=17)$ recovered uneventfully from the surgeries, and appeared normal throughout the remaining course of the study (12 weeks). There was no indication of hernia or adhesions in the test animals. Macroscopically the closed sites showed no abnormalities, and wound healing was not disturbed. Histological examination of the tissue at the surgical incision lines showed progressive healing with no evidence of wound dehiscence at any time during the study. 


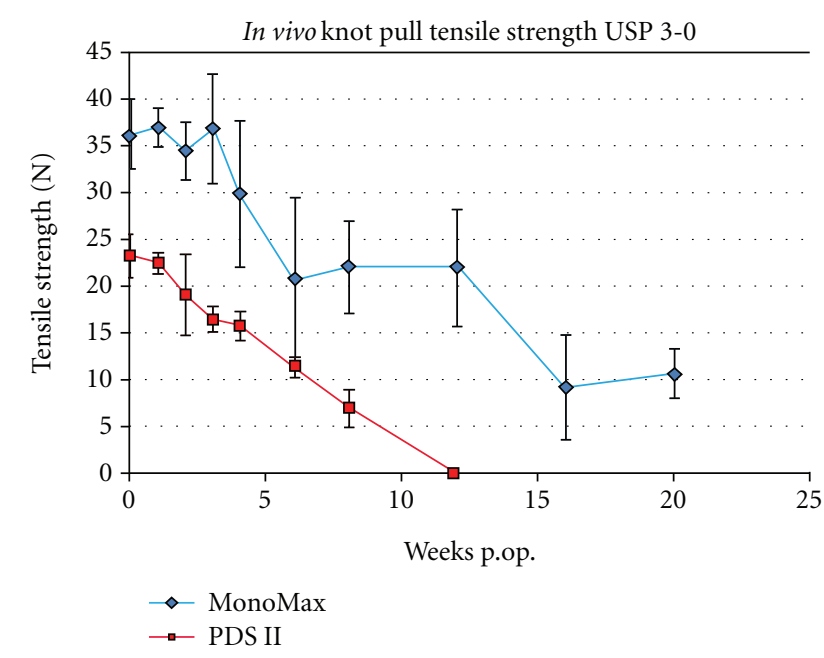

Figure 6: In Vivo Knot Pull Tensile Strength (KPTS) degradation profile of a $\mathrm{P} 4 \mathrm{HB}$ monofilament and PDS II suture size 3-0 implanted subcutaneously in White New Zealand rabbits up to 20 weeks.

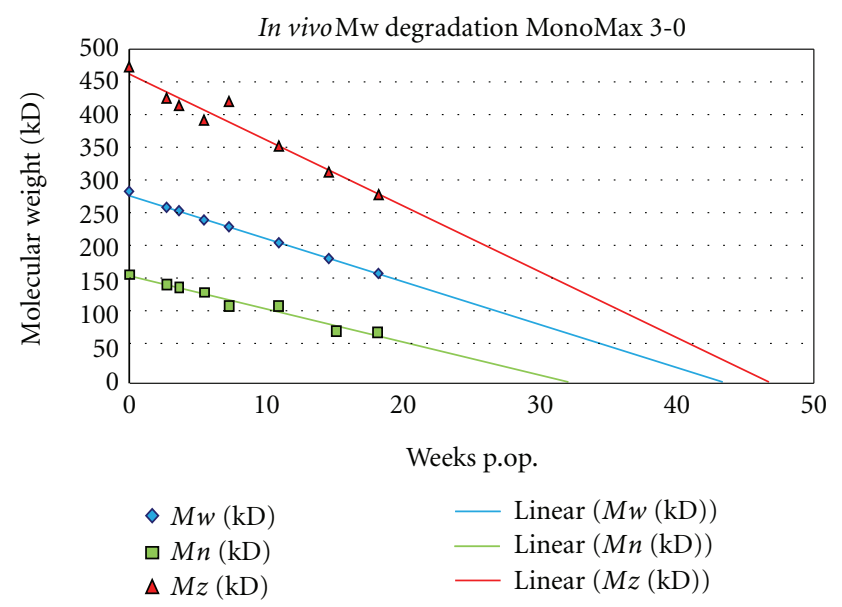

Figure 7: In Vivo molecular weight degradation of a $\mathrm{P} 4 \mathrm{HB}$ monofilament fiber size 3-0 implanted subcutaneously in White New Zealand rabbits up to 20 weeks.

\section{Discussion}

MonoMax suture fiber was prepared by melt extrusion of poly-4-hydroxybutyrate homopolymer for preclinical evaluation as a new absorbable monofilament suture. The properties of the MonoMax suture were compared to PDS II, another monofilament suture material, in order to identify the possible clinical benefits of this new biomaterial.

As shown in Table 2, the MonoMax suture has comparable tensile and knot strength to the PDS II suture material however, the MonoMax suture is significantly more pliable and flexible than the PDS II suture or any of the other absorbable monofilaments tested. These properties can be quantified by measuring the value of the Young's modulus and the bending force of the respective sutures. As far as the Young's modulus is concerned, the value of MonoMax suture is about one-third the value of the PDS II suture fiber. Even compared to the hard segment-soft segment type sutures like Monocryl and Biosyn, the value of MonoMax monofilament ranges between one-half to one-third the values of these sutures. The lower Young's modulus contributes to the very good knot security of the MonoMax monofilament by providing a more flexible suture with a softer surface resulting in a superior interlocking of the fiber that results in a very good knot safety factor. Consequently, a lower number of knot throws is needed to make a secure knot, resulting in less irritating mass and potentially less foreign body reaction. Furthermore the MonoMax fiber's intrinsic elasticity allows the surgeon to apply more tension to the knot during tying.

The low bending stiffness value of the MonoMax fiber makes the suture extremely pliable and flexible for surgical manipulation, which is advantageous when the surgeon uses suture techniques that include close radii. The MonoMax suture material shows little memory and its fiber ends are less irritating than for a stiffer suture fiber. This is of some importance in situations when for instance the surgeon cuts the fiber a short distance from the knot and has to cover the fiber ends in the surrounding tissues.

The strength retention of the MonoMax suture was evaluated both in vitro and in vivo. In vitro, the MonoMax monofilament was found to be less susceptible to hydrolysis than currently available suture materials, giving more safety to slow-healing tissues. The MonoMax suture is in fact fairly resistant to neutral conditions and maintains strength beyond 12 weeks. Accelerated degradation can be achieved under more severe acidic conditions. Figure 4 shows the accelerated degradation profile of a size 3-0 MonoMax suture exposed to $3 \mathrm{M}$ hydrochloric acid for 48 hours. In $3 \mathrm{M} \mathrm{HCl}$, the suture loses approx. $50 \%$ of its initial tensile strength within one day and is $90 \%$ degraded after 2 days. Figure 6 shows the in vivo strength retention of a size 30 MonoMax monofilament implanted in the subcutaneous tissue of rabbits. The MonoMax fiber undergoes a relatively slow degradation process in vivo. As the measurements from the implanted size 3-0 suture demonstrate, the MonoMax monofilament fiber retains about $50 \%$ of its initial tensile strength after 3 months, which is a significantly slower degradation profile than for other commercially available absorbable monofilaments (Maxon 50\% BSR after 28 days, PDS II after 35 days). Therefore, the MonoMax fiber may be an excellent choice for surgical indications involving the apposition of slow healing tissues such as fascia or tendons when an application of a nonabsorbable suture is not appropriate.

The biocompatibility of the MonoMax suture was demonstrated in a variety of in vitro and in vivo biocompatibility testing. Histological examination of the MonoMax monofilament size 3-0 fiber implanted in the dorsal muscle of rats up to 64 weeks revealed again the very good biocompatibility of the MonoMax suture without any adverse effects. First signs of phagocytosis of the suture were visible after 16 weeks, and at 32 weeks, the fiber showed a surface degradation with macrophages eventually penetrating the whole fiber diameter. After 50 weeks, the fiber was replaced 


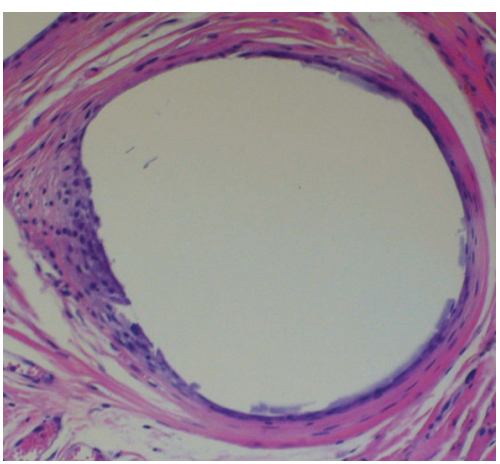

(a) 8 weeks p.op.

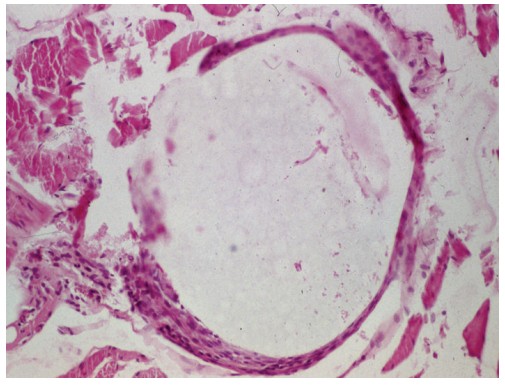

(c) 50 weeks p.op.

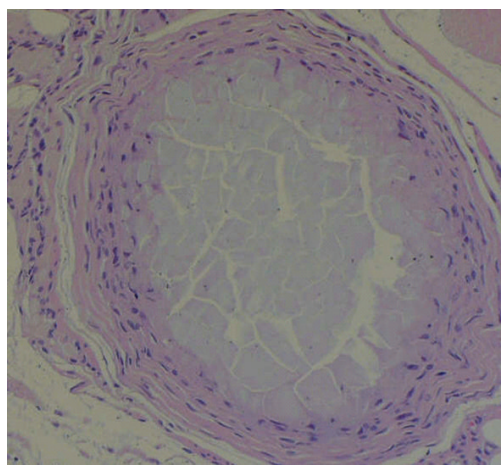

(b) 32 weeks p.op.

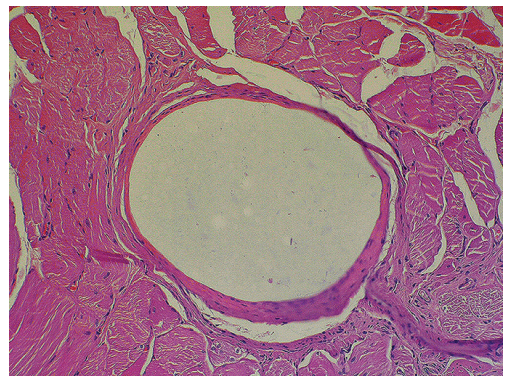

(d) 64 weeks p.op.

Figure 8: Histology images of EtO sterilized P4HB monofilament size 3-0 implanted in the long. dorsi j. of male Wistar rats.

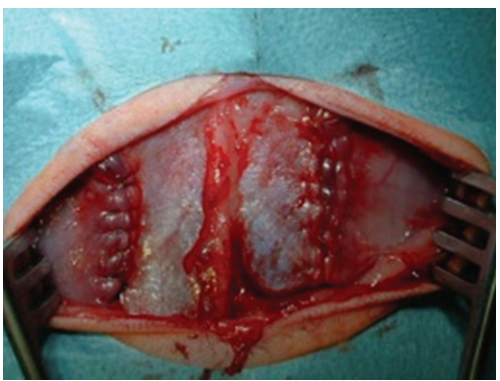

(a)

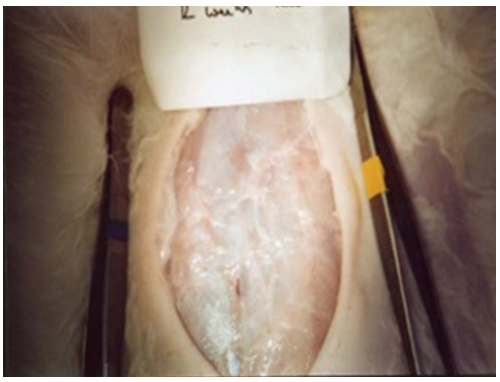

(c)

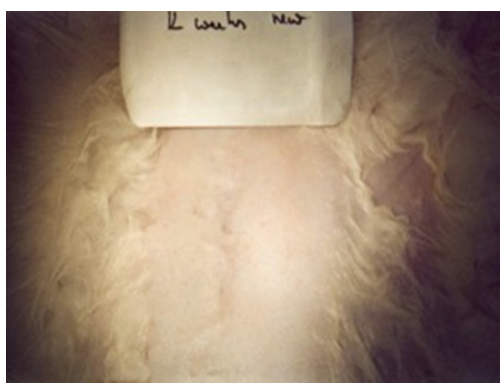

(b)

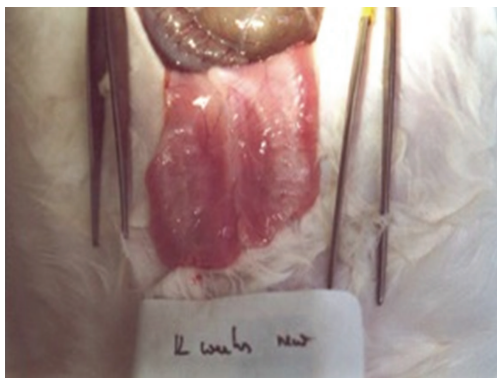

(d)

FIGURE 9: Implantation of P4HB and PDS II sutures in a rabbit model to evaluate surgical functionality. Full thickness abdominal incisions were closed with PDS II (left) or poly-4-hydroxybutyrate (right) sutures. (a) at implantation. (b) skin incision line at 12 weeks. (c) laparotomy suture line (skin side) at 12 weeks. (d) laparotomy suture line (intestinal side) at 12 weeks. 
by fine granular material, leading to an essential mass absorption after 64 weeks.

Finally, the surgical functionality of the suture was demonstrated in a rabbit model for abdominal wound closure. The MonoMax suture performed well and no instances of wound dehiscence, hernias, or adhesions were found. There was also no evidence of tissue irritation or infections.

\section{Conclusion}

MonoMax suture, a new monofilament suture based on the poly-4-hydroxybutyrate homopolymer, has been developed that combines a high tensile strength with the highest pliability and the lowest Young's modulus of any commercially available monofilament absorbable suture. In addition, this suture offers a number of clinical benefits to meet unmet needs, including prolonged strength retention, low tissue drag, and knot security.

The biocompatibility of the MonoMax suture was evaluated for cytotoxicity, irritation, sensitization, acute systemic toxicity, pyrogenicity, genotoxicity, subchronic systemic toxicity, and chronic toxicity, and tissue reaction was assessed by intramuscular implantation. All tests indicated that the MonoMax suture presents an excellent biocompatibility and physiologically is well integrated in the tissues. Absorption of a size 3-0 suture was found to be substantially complete at about 64 weeks.

A surgical functionality study was undertaken to evaluate the performance of the MonoMax suture in a rabbit model of abdominal soft tissue approximation. Full thickness abdominal incisions were successfully and satisfactorily repaired with the MonoMax suture without causing tissue irritation or infections. The properties of this suture material were demonstrated in a human clinical trial for the abdominal wall closure (ISSAAC Trial) [21] and the suture was found to be safe for abdominal wall closure after midline laparotomy [22].

Considering the excellent properties of the MonoMax suture, it could be especially useful as a suture material for slowly healing tissues. We expect this new suture to offer surgeons an alternative to faster degrading absorbable sutures or permanent nonabsorbable materials when longterm wound support is desired.

\section{Acknowledgment}

Tepha, Inc. would like to acknowledge NIH SBIR Grant no. 2 R44 EB003644-02 for partial support of this work.

\section{References}

[1] M. K. Hirko, P. H. Lin, H. P. Greisler, and C. C. Chu, "Biological properties of suture materials," in Wound Closure Materials, C. C. Chu, J. A. Von Fraunhofer, and H. P. Greisler, Eds., pp. 237-287, CRC Press, Boca Raton, Fla, USA, 1996.

[2] D. P. Martin and S. F. Williams, "Medical applications of poly4-hydroxybutyrate: A strong flexible absorbable biomaterial," Biochemical Engineering Journal, vol. 16, no. 2, pp. 97-105, 2003.
[3] Y. Doi, Microbial Polyesters, VCH, New York, NY, USA, 1990.

[4] S. F. Williams and D. P. Martin, "Applications of PHAs in Medicine and Pharmacy," in Biopolymers, Y. Doi and A. Steinbüchel, Eds., pp. 91-127, Wiley-VCH, 2002.

[5] Q. Wu, Y. Wang, and G. Q. Chen, "Medical application of microbial biopolyesters polyhydroxyalkanoates," Artificial Cells, Blood Substitutes, and Biotechnology, vol. 37, no. 1, pp. $1-12,2009$.

[6] G. W. Huisman, F. Skraly, D. P. Martin, and O. P. Peoples, "Biological systems for manufacture of polyhydroxyalkanoate polymers containing 4-hydroxyacids," US Patent no. $6,316,262$.

[7] D. E. Dennis and H. E. Valentin, "Methods of making polyhydroxyalkanoates comprising 4-hydroxybutyrate monomer units," US Patent no. 6,117,658.

[8] Y. Hori, A. Yamaguchi, and T. Hagiwara, "Chemical synthesis of high molecular weight poly(3-hydroxybutyrate-co-4hydroxybutyrate)," Polymer, vol. 36 , no. 24 , pp. 4703-4705, 1995.

[9] K. N. Houk, A. Jabbari, H. K. Hall, and C. Alemán, "Why $\delta$-valerolactone polymerizes and $\gamma$-butyrolactone does not," Journal of Organic Chemistry, vol. 73, no. 7, pp. 2674-2678, 2008.

[10] "Biological evaluation of medical devices - Part 7: Ethylene oxide sterilization residuals," ISO 10993-7:2008.

[11] The United States Pharmacopeia, The National Formulation USP 32-NF 27 (2010), chapter 881 Tensile Strength: 380, United States Pharmacopeial Convention INC. Rockville, Md, USA.

[12] European Pharmacopeia, The European Directorate for the Quality of Medicines \& HealthCare, Strasbourg, France, 6th edition, 2010.

[13] H. Tera and C. Aberg, "Tensile strengths of twelve types of knot employed in surgery, using different suture materials," Acta Chirurgica Scandinavica, vol. 142, no. 1, pp. 1-7, 1976.

[14] "Biological evaluation of medical devices - Part 1: Evaluation and testing," ISO 10993-1:2003.

[15] R. S. Bezwada, D. D. Jamiolkowski, I. Y. Lee et al., "Monocryl suture, a new ultra-pliable absorbable monofilament suture," Biomaterials, vol. 16, no. 15, pp. 1141-1148, 1995.

[16] G. T. Rodeheaver, K. A. Beltran, C. W. Green et al., "Bio-mechanical and clinical performance of a new synthetic monofilament absorbable suture," Journal of Long-Term Effects of Medical Implants, vol. 6, no. 3-4, pp. 181-198, 1996.

[17] T. Nelson, E. Kaufman, J. Kline, and L. Sokoloff, "The extraneural distribution of $\gamma$-hydroxybutyrate," Journal of Neurochemistry, vol. 37, no. 5, pp. 1345-1348, 1981.

[18] S. L. Sendelbeck and C. L. Girdis, "Disposition of a 14Clabeled bioerodible polyorthoester and its hydrolysis products, 4-hydroxybutyrate and cis,trans-1,4-bis(hydroxymethyl)cyclohexane, in rats," Drug Metabolism and Disposition, vol. 13, no. 3, pp. 291-295, 1985.

[19] National Toxicology Program (NTP), "NTP Summary report on the metabolism, disposition and toxicity of 1,4butanediol," Toxicity Report 54, NIH Publication no. 96-3932, 1996.

[20] National Toxicology Program (NTP), "Toxicology and carcinogenesis studies of gamma-butyrolactone (CAS No. 9648-0) in F344/N rats and B6C3F1 mice (gavage studies)," Technical Report 406, NIH Publication no. 92-3137, 1992.

[21] L. Fischer, P. Baumann, J. Hüsing et al., "A historically controlled, single-arm, multi-centre, prospective trial to evaluate the safety and efficacy of MonoMax suture material for abdominal wall closure after primary midline laparotomy. 
ISSAAC-Trial [NCT005725079]," BMC Surgery, vol. 8, article 12, 2008.

[22] M. Albertsmeier, C. M. Seiler, L. Fischer et al., "Evaluation of the safety and efficacy of MonoMax suture material for abdominal wall closure after primary midline laparotomy-a controlled prospective multicenter trial: ISSAAC [NCT005725079]," Langenbeck's Archives of Surgery, vol. 397, pp. 363-371, 2012. 

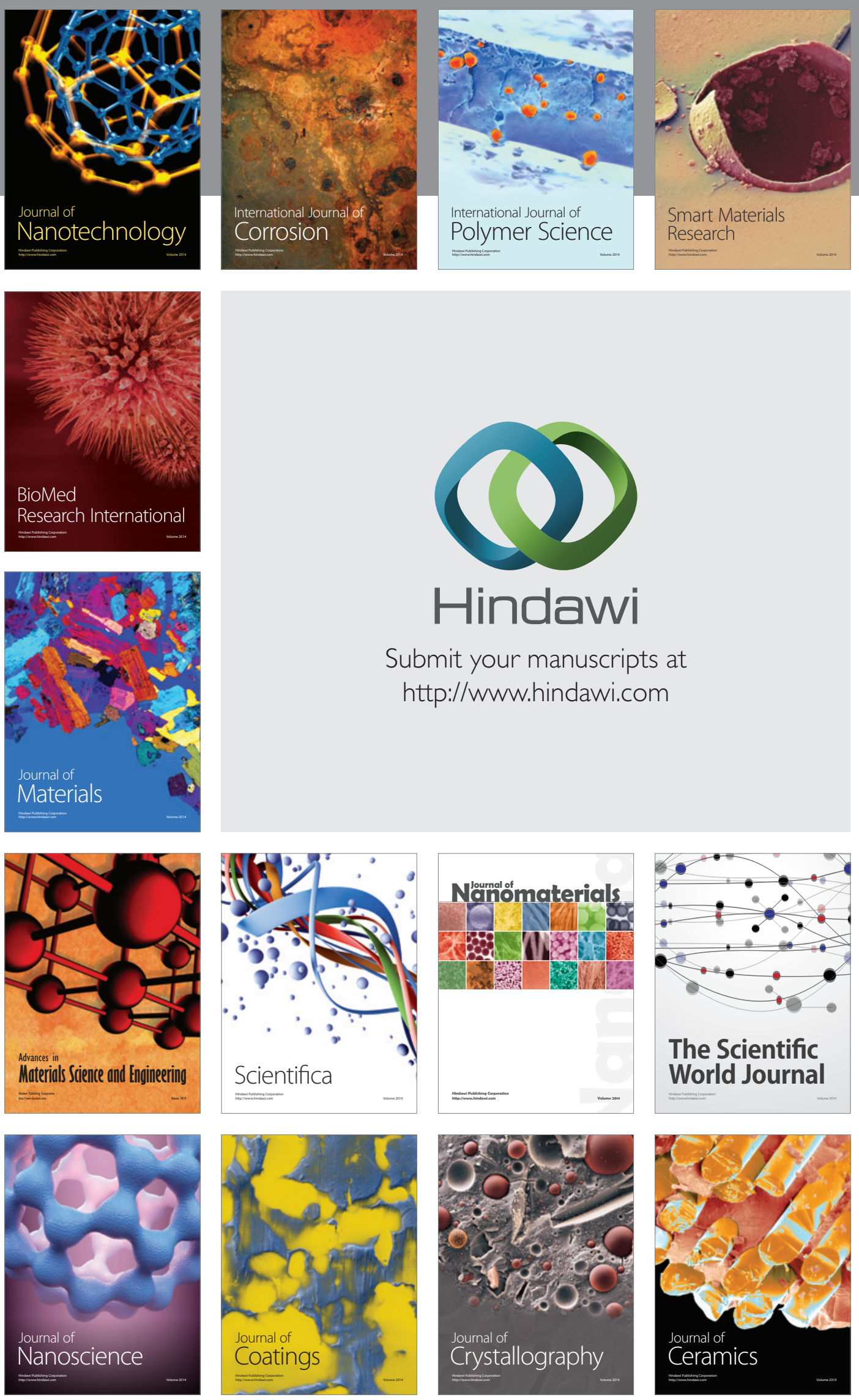

The Scientific World Journal

Submit your manuscripts at

http://www.hindawi.com

\section{World Journal}

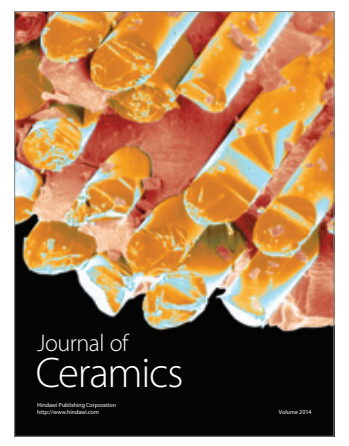

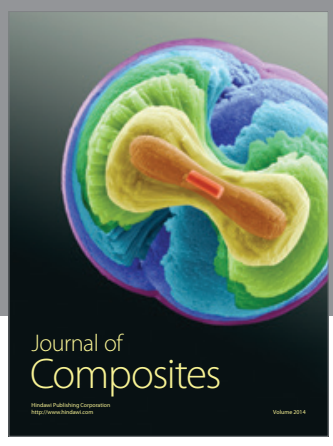
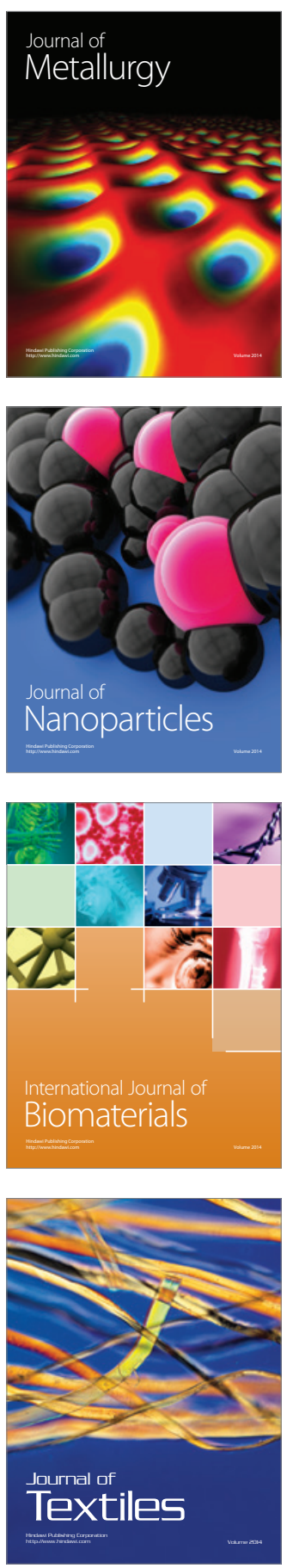